\title{
Clinical trials with MRNA electroporated dendritic cells for stage III/IV melanoma patients
}

\author{
Sofie Wilgenhof ${ }^{1}$, Jurgen Corthals ${ }^{1}$, Carlo Heirman', Bart Neyns², Kris Thielemans ${ }^{3^{*}}$ \\ From 30th Annual Meeting and Associated Programs of the Society for Immunotherapy of Cancer (SITC \\ 2015) \\ National Harbor, MD, USA. 4-8 November 2015
}

\section{Background}

TriMixDC-MEL consists of autologous monocyte-derived DC that are electroporated with synthetic mRNA encoding CD40 ligand, a constitutively active TLR4, CD70 and fusion proteins of DC.LAMP with 4 melanoma associated antigens (MAGE-A3, MAGE-C2, tyrosinase and gp100).

\section{Methods}

TriMixDC-MEL was investigated in patients (pts) with pretreated advanced melanoma, either as a single agent (Phase Ib; NCT01066390) or combined with IPI (Phase II; NCT01302496; $10 \mathrm{mg} / \mathrm{kg}$ q3wks x4) and also in melanoma pts who are disease free following local treatment of macrometastases. TriMixDC-MEL was administered by the IV and ID-route (4 to 5 admin; $4.10^{6}$-ID/20.10 - -IV).

\section{Results}

In pts with unresectable AJCC stage III or IV melanoma, respectively 15 and 39 pts were treated in the Phase Ib and the -II trial. DC-related AEs consisted of local inflammatory skin reactions at the DC-injection site (all pts), grade (gr) 2 acute post-IV injection chills in $20 \%$ and $38 \%$, and gr 1-2 flu-like syndrome in $53 \%$ and $85 \%$ of pts treated respectively with DC or DC+IPI. Grade 3 or 4 irAEs occurred in 36\% of DC+IPI treated pts. ORR for DC: 27\% (2 CR, 2 PR; 3 are ongoing after $>+51 \mathrm{mths}$ ) and $38 \%$ for DC+IPI ( 8 CR, 7 PR; 8 are ongoing after $>+16$, mths). Median PFS and OS are respectively 5 (95\% CI $0-10)$ and $14 \mathrm{mths}(95 \% \mathrm{CI}$ 5-23) for DC and 6.2 (95\% CI 2-10) and 13 mths (95\% CI, 9-18) for DC+IPI. The 1, 2 and 3y OS\% for DC+IPI were: $59 \%$ (95\% CI 43-74), 38\% (95\% CI, 23-53), and 34\% (95\% CI, 19-50). Treatment with TriMixDC-MEL, especially in combination with IPI, is tolerable and results in a high rate of durable tumor responses. In the adjuvant setting (NCT01676779), 41 patients were randomized between the TriMixDC-MEL treatment arm $(n=21)$ and control-arm $(n=20)$. Baseline characteristics were well balanced between both groups. After a median follow-up of $18 \mathrm{mths}$ (range 5 to $30 \mathrm{mths}) 20$ patients experienced a non-salvageable melanoma recurrence (6 on the DC- and 14 on the control-arm). The rate of patients who were disease-free at 1 year (evaluable population $=35$ patients) was higher in the TriMixDC-MEL treated group (65\% [95\%CI 42-87] vs. 34\% [13-55]). TriMixDC-MEL was well tolerated (no grade $>3 \mathrm{AE}$ ).

\section{Conclusions}

The results of this non-comparative randomized controlled Phase II clinical trial of TriMixDC-MEL ID/IV versus observation support the further evaluation of TriMixDCMEL as a well-tolerated adjuvant therapy for melanoma patients following the resection of macrometastases.

\section{Authors' details \\ ${ }^{1}$ Vrije Universiteit Brussel, Brussels, Belgium. ${ }^{2}$ Universitair Ziekenhuis Brussel, Brussels, Belgium. ${ }^{3}$ Vrije Universiteit Brussels, Brussels, Belgium.}

Published: 4 November 2015

doi:10.1186/2051-1426-3-S2-P211

Cite this article as: Wilgenhof et al:: Clinical trials with MRNA

electroporated dendritic cells for stage III/IV melanoma patients. Journal for ImmunoTherapy of Cancer 2015 3(Suppl 2):P211.

${ }^{3}$ Vrije Universiteit Brussels, Brussels, Belgium

Full list of author information is available at the end of the article 